\title{
CIGTF Enhanced Precision Reference Systems
}

\author{
Robert S. Lawrence, George Gregory, Derryl Stutz, \\ Jerry Sanchez, Brent Neal \\ 746th Test Squadron, Holloman AFB, NM
}

\begin{abstract}
The 746th Test Squadron at Holloman AFB has developed and utilized the Central Inertial Guidance Test Facility (CIGTF) High Accuracy Post-processing Reference System (CHAPS). CHAPS is a multi-sensor navigation reference system used to evaluate position, velocity, and attitude performance of Global Positioning System (GPS), Inertial Navigation System (INS), and Embedded GPS/INS (EGI) navigation systems on large vehicles and aircraft. Reference data is processed post-test with accuracy ranges from a meter to sub-meter depending on the reference configuration and test environment (profile, trajectory dynamics, GPS jamming, etc.). The GPS Aided Inertial Navigation Reference (GAINR) system developed by the Air Force Flight Test Center (Edwards AFB) offered other utilization capabilities (test beds and post-processing time). The basic sensor assembly is an EGI navigation system. The data are post-processed with Multisensor Optimal Smoothing Estimation Software (MOSES). Incorporating CHAPS and GAINR capabilities generates a reference system with enhanced accuracy (sub-meter) in a dynamic GPS non-jamming/jamming environment. This paper will present the enhanced reference system combination of CHAPS/GAINR capabilities, characterization process and development methodology.
\end{abstract}

\section{KEYWORDS}

Reference System, Instrumentation, Data Acquisition System, Embedded Navigation System

\section{INTRODUCTION}

The 46th Test Group CIGTF, officially known as the 746th Test Squadron, established in May 1959 , is the world's premier facility for system/component testing of missile guidance and control systems, aircraft INS, GPS and EGI navigation systems. The inertial laboratory consists of a variety of test beds; centrifuges, two and three axis test tables, rotary and linear vibrators, environment chambers, and seismically stable platforms. The GPS Navigation Test and Evaluation Laboratory (NavTEL) consists of a variety of GPS simulators supporting the test and integration of GPS receivers, antennas, INS's and EGI systems. In conjunction with laboratory testing, field testing (van, flight and sled track) provides an operational test environment with accurate references to perform comprehensive quantitative analysis.

In the early 1970's, the Completely Integrated Reference Instrumentation System (CIRIS) was the van and flight test reference system (10-meter accuracy), designed to evaluate unaided inertial navigation systems. In the early 1990's the CIGTF High Accuracy Post-processing 
Reference System (CHAPS) was the replacement reference with significantly improved accuracy (sub-meter) and performance in a GPS electronic countermeasures (ECM) jamming environment. With the evolution of design enhancements, and future test requirements, the GPS Aided Inertial Navigation Reference (GAINR) system developed by the Air Force Flight Test Center (Edwards AFB) offered other advanced capabilities, specifically EGI utilization. Both reference systems, offering unique capabilities to accommodate specific test requirements, were upgraded for future requirements, and combined creating the present CIGTF Reference System (CRS).

The objective of the paper is to present the enhanced reference system combination of CHAPS/GAINR capabilities, characterization process and development methodology; using PC104 technology to accomplish the data acquisition and control tasks.

\section{CHAPS DESCRIPTION}

CHAPS, operational 1996, is a rack-mounted loosely integrated reference system, consisting of navigation sensor components, data acquisition system and post-mission data processing software. The VME-based data acquisition computer controlled and collected measurements from; SNU-84 INS, Rockwell-Collins Miniature Airborne GPS Receiver (MAGR), Ashtech Z12 carrier phase GPS receiver, two Cubic CR-100 range/range rate interrogators/transponder system (RRS) and altitude an encoder. The post-mission processing mechanization utilized INS, differential GPS and RRS measurements in an extended Kalman filter/smoother algorithm. The loosely integrated configuration enhances the navigation sensor interchangeability, specifically INS performance, which quantifies velocity accuracy. Nominal performance accuracies of the reference trajectory characterized for specific environments are:

1. (sub-meter) low-to-medium dynamics

2. (meter) high dynamics (hardware/space limited)

3. (meter) GPS jamming

\section{GAINR DESCRIPTION}

GAINR is a rack-mounted and standalone loosely/tightly integrated reference system consisting of an EGI, additional navigation sensors, data acquisition system and post-mission data processing software. A DOS-based computer collects measurements from the reference/EGI navigation system. The tightly integrated raw EGI measurements are post processed with the Multi-sensor Optimal Smoothing Estimation Software (MOSES) providing a reference trajectory and sensor error estimates. Additional measurements are collected from the RRS and Ashtech Z12 carrier phase GPS receiver. The additional reference trajectories are generated utilizing the loosely integrated EGI inertial, differential GPS and RRS measurements in an extended Kalman filter/smoother algorithm. These additional trajectories and MOSES trajectories are combined using a Federated Filter algorithm, improving specific test scenario accuracy. Nominal performance accuracies of the reference trajectory characterized for specific environments are:

1. (sub-meter) low-to-medium dynamic

2. (meter) high dynamics

3. (meter) GPS Jamming 


\section{CRS DESCRIPTION}

CRS is a rack mounted (Figure 1) and standalone, loosely/tightly integrated system, consisting of navigation sensors/subsystems, data acquisition system (DAS) and post-mission processing mechanization (Figure 2). The DAS, DOS-based PC-104 technology, computer performs the primary functions of data collection and real time control for the following subsystems: [1] EGI navigation system, [2] GPS receiver/receivers, [3] INS and [4] RRS. Other subsystems supported in the CRS architecture are the GPS environment measurement system (GEMS), data link, altitude encoder and Satellite Reference Station (SRS) receiver, supporting differential GPS (DGPS) algorithms. The post-mission processing mechanization utilizes combinations of the subsystem measurements in an extended Kalman filter/smoother algorithm to produce an optimal reference trajectory. Nominal performance accuracies of the reference trajectory characterized for specific environments are:

1. (sub-meter) low-to-high dynamic

2. (meter) GPS jamming

Characterization results will quantify accuracy estimates, depending on the configured subsystems.

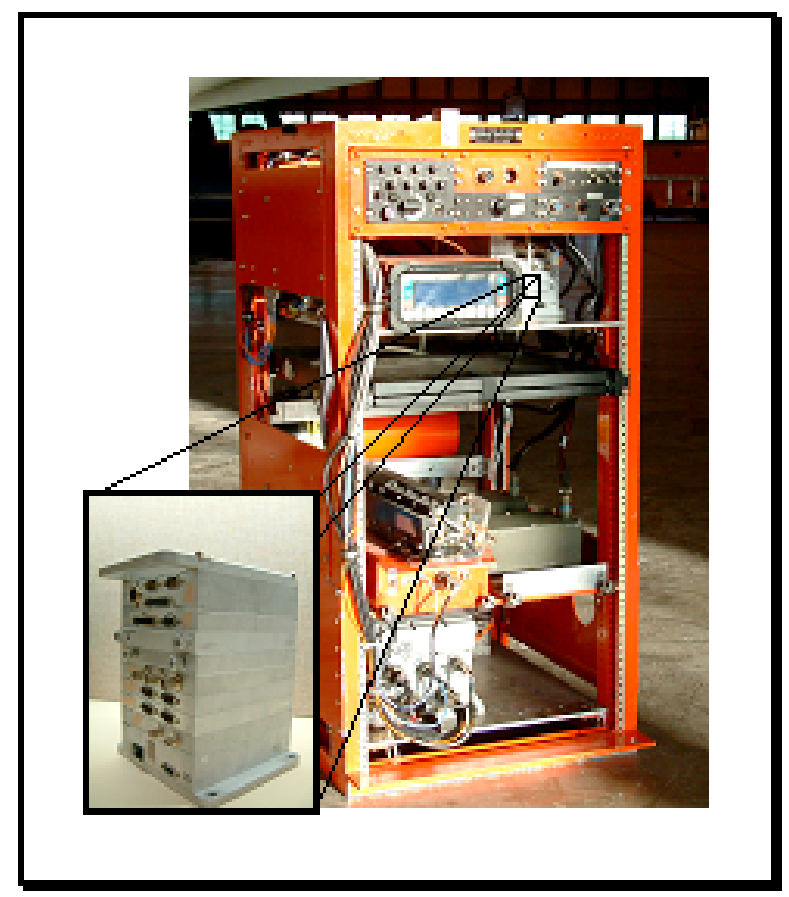

Figure 1: CRS

[1] GPS/INS Subsystem (EGI): The sensor assembly is a Honeywell H-764GU Embedded GPS/INS (EGI) Navigation system modified to produce high rate $(256 \mathrm{~Hz})$ gyro $(\Delta \theta)$ and 
accelerometer $(\Delta v)$ measurements. A Rockwell Collins GEM IV all-in-view receiver provides GPS satellite pseudo-range, delta-range and carrier-phase data. The inertial sensor assembly incorporates the Honeywell Digital Laser Gyros. In addition to the raw measurement data, inertial, GPS and blended GPS/INS solutions are available on the 1553 bus interface.

[2] GPS Receiver Subsystem (DGPS): The GPS receiver is normally a commercial 12-channel dual frequency carrier phase Ashtech Z-12. The L1-L2 P-code is tracked using a P-W codeless technology. The SRS Z-12 receiver provides differential code and carrier phase corrections. Post-processing software, Precision DGPS Navigation / Survey Software (PNAV), combines the two measurements to form a fixed-ambiguity carrier phase or code (pseudo-range) differential GPS (DGPS) position vector at a 1 and $10-\mathrm{Hz}$ rate.

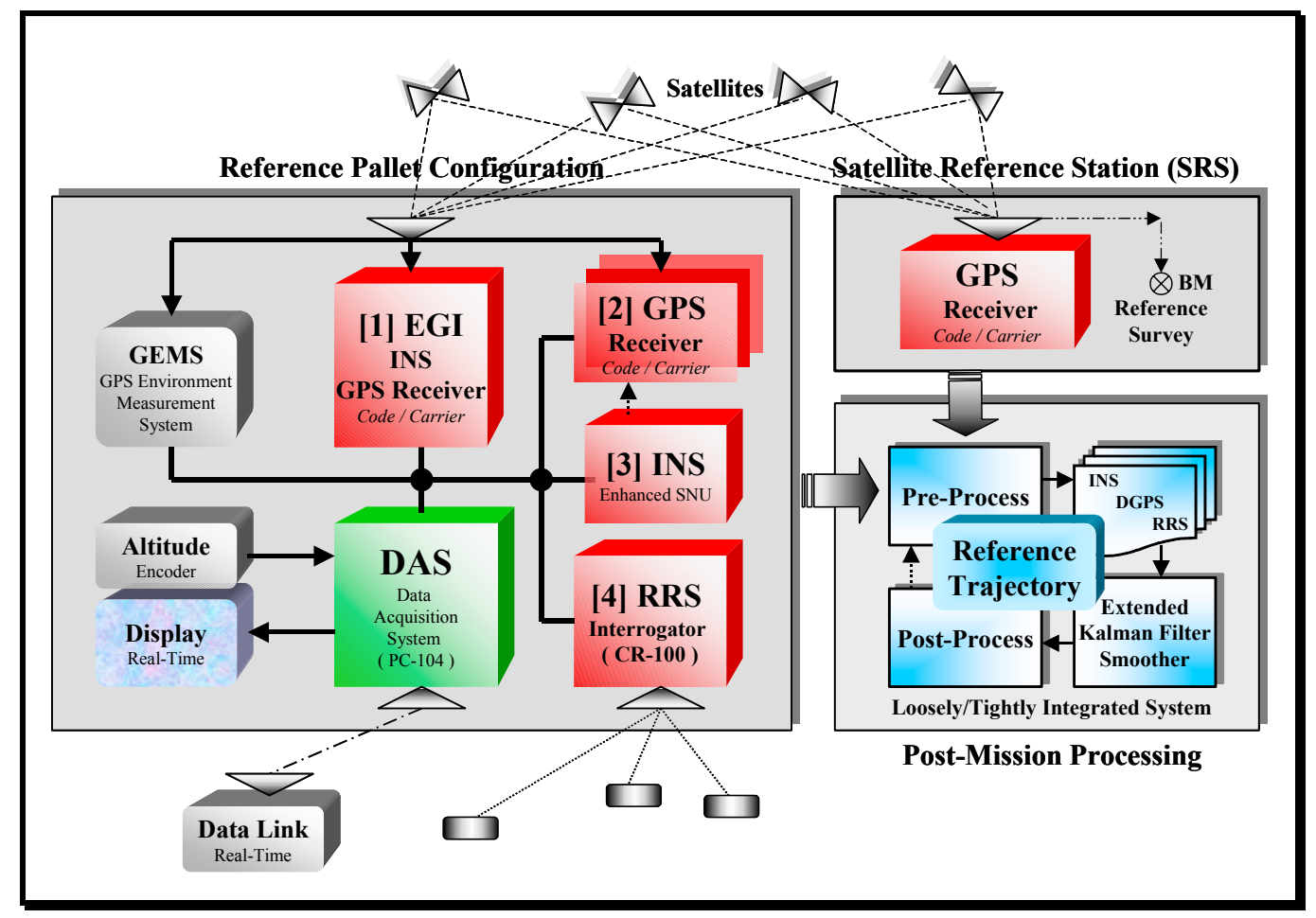

Figure 2: CRS Architecture

[3] Inertial Navigation Subsystem (INS): The inertial navigation system is a Honeywell H423 strapdown ring laser gyro system, which complies with the USAF standard medium accuracy $(0.8 \mathrm{~nm} / \mathrm{hr})$ navigation unit specification, SNU-84-1. A modification needed for reference velocity requirements utilizes the enhanced SNU-84 (ESNU), providing increased position accuracy $(0.1 \mathrm{~nm} / \mathrm{hr})$. Altitude aiding of the INS is performed using a combination of GPS and baro-altimeter altitude.

[4] Range and Range-Rate Subsystem (RRS): The CR-100 RRS consists of multiple groundbased transponders and one or more interrogators. The transponders are portable and are arranged to provide good geometry throughout the aircraft flight profile. Up to 255 different transponder identifications are available, although a typical flight test uses only 20-50 different transponders. 
The interrogator transmits and receives on one of three channels in the 2325 to $2412 \mathrm{MHz}$ range. Since this frequency band is much higher than the GPS L1 and L2 frequencies, the interrogator can continue to collect measurements in the presence of GPS jamming.

The range measurement is normally accurate to within 1-2 meters including atmospheric delays, survey errors and calibration errors. The "range-rate" measurements are actually delta-ranges where the change in carrier phase is tracked over a short integration interval. The resulting lineof-sight (LOS) range rate is accurate to within $0.03 \mathrm{~m} / \mathrm{sec}$.

GPS Environment Measurement Subsystem (GEMS): The GEMS measures real-time GPS jamming signal levels, specifically the GPS Jam-to-Signal $(\mathrm{J} / \mathrm{S})$ ratio.

Data Acquisition System (DAS): The DAS, using PC-104 technology (Figure 3), functions as the control/monitor/collect component of CRS architecture to tie all the subsystems together as a hybrid reference system. Software performs all initialization functions and records navigation sensor data to the 1 Gbyte Flashdisk. All timing is referenced to GPS UTC time using Havequick data from the EGI.

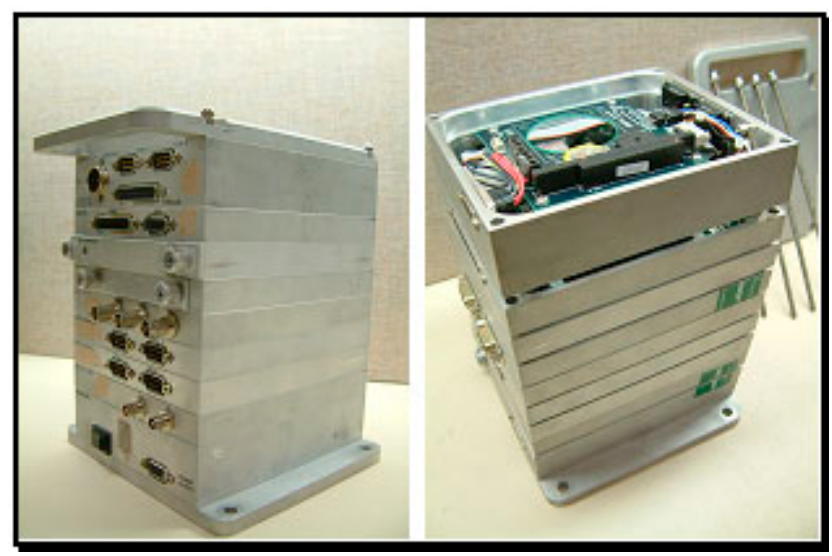

Figure 3. DAS Hardware

The DAS is configured using the following modular PC-104 cards:

1. Real Time Devices (RTD) $100 \mathrm{~W}$ power supply

2. RTD Am486DX100 based Computer Processing Unit (CPU)

3. RTD VGA video card

4. RTD ATA Flashdisk card

5. RTD asynchronous serial cards

6. SBS MIL-STD 1553B card

7. JX12 PC-104SG synchronizable clock card.

The DAS software is written in C language running under the DOS 6.22 operating system, supporting data requirements demanding a real-time system with deterministic and preemptive scheduling guarantees. The $\mathrm{C}$ general-purpose language, well suited for embedded applications, 
is widely used, concise and features high-level control flow as well as low-level access to the computer hardware.

\section{POST-MISSION PROCESSING}

The post-mission mechanization (Figure 4), loosely/tightly integrated configuration, utilizes processed combinations of the subsystem measurements in an extended Kalman filter/smoother algorithm, generating the optimal reference trajectory. The Kalman filter is a nonlinear aided INS navigation filter; of which the INS output serves as the indicated (nominal) trajectory and GPS/RRS measurement vectors are processed to estimate position, velocity, attitude and other errors. The smoother, classification fixed-interval, generates smoother estimates, from apriori filter error estimates, covariance and model dynamics. The smoother estimated errors are then subtracted from the indicated trajectory to produce the reference trajectory, position, velocity and attitude. The error models for the INS, GPS and RRS subsystems are additional states, depending upon the mission subsystem configuration.

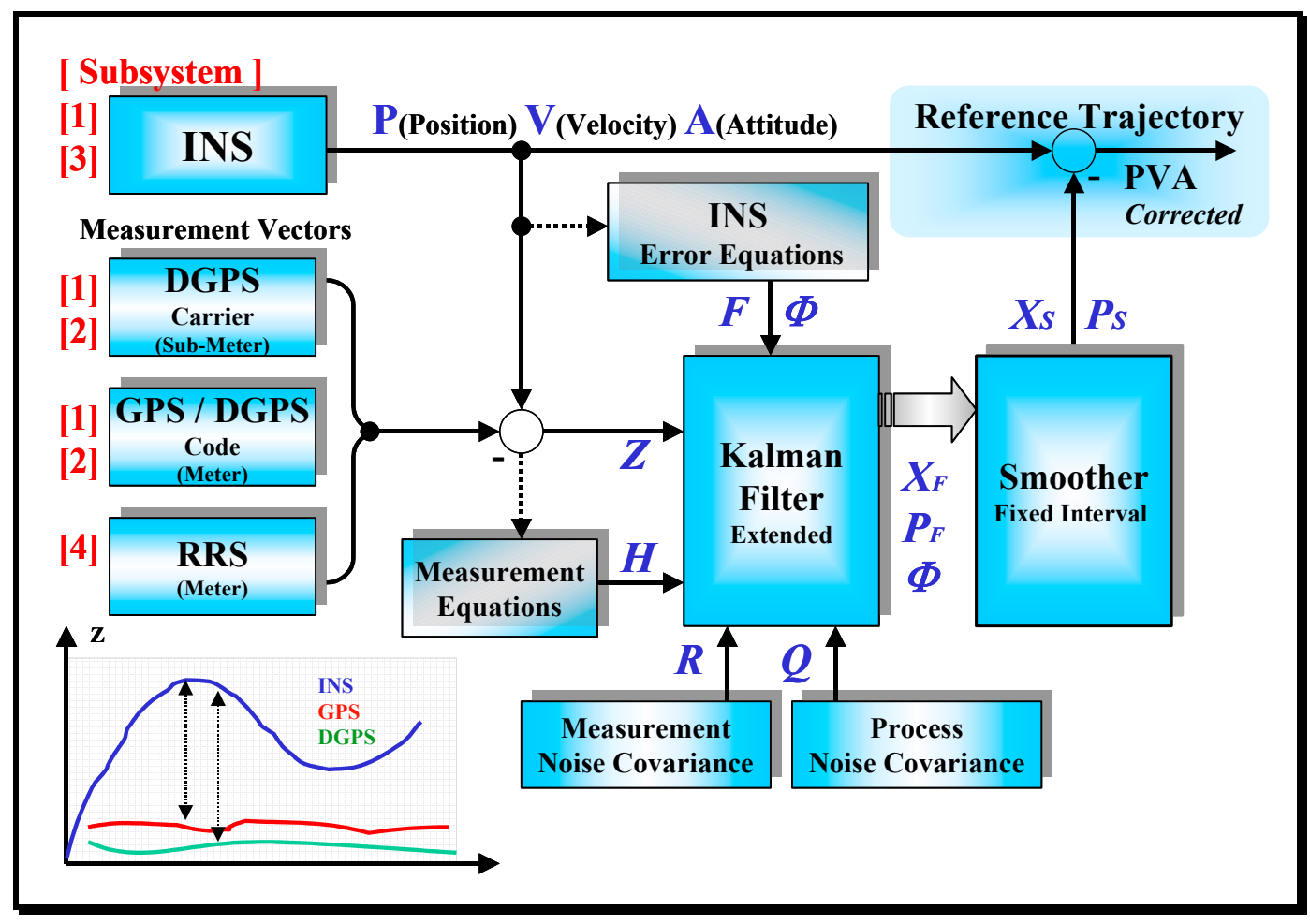

Figure 4: Processing Mechanization

\section{CHARACTERIZATION RESULTS}

The Holloman High-Speed Test Track (Figure 5) was the primary test bed for the characterization of performance accuracy. The test track, spanning a linear distance of 15,480 meters, is uniquely applicable for testing strategic guidance systems and components. Providing 
controllable profile dynamics and an accurate reference system (Position: $<0.001 \mathrm{~m}$, Velocity: $<0.0005 \mathrm{~m} / \mathrm{sec}$, Azimuth: $<0.5 \mathrm{arcsec}$ ) permitted comprehensive quantitative analysis. Track tests consisted of low velocity $(8$-to- $16 \mathrm{~m} / \mathrm{sec})$ recovery support vehicle tow tests and variable medium velocity $(\approx 160 \mathrm{~m} / \mathrm{sec}, 3 g$ acceleration) rocket sled tests. Van and flight tests provided the characterization of operational performance for low (Figure 6), medium (Figure 7), and high (Figure 8) dynamics, depending on the scenario/environment and subsystem utilization or availability.

CHAPS performance was characterized with an extensive test series (sled track, van and flight) under optimum and degraded conditions (loss of transponder and/or differential corrections). GAINR performance was characterized with a test series in conjunction with CHAPS consisting of sled track (low velocity), van and flight. CRS performance accuracy, quantified from CHAPS and GAINR configurations, and additional characterization tests, according to the subsystem configuration selected (Figure 2), is detailed in the position and velocity accuracy estimates (Table 1).

Table 1: CRS Subsystem Configuration Accuracy

\begin{tabular}{|c|c|c|c|c|c|}
\hline \multirow{2}{*}{\multicolumn{3}{|c|}{$\begin{array}{l}\text { [ Subsystem ] } \\
\text { Configuration }\end{array}$}} & \multicolumn{3}{|c|}{ RMS Position (m) } \\
\hline & & & \multirow{2}{*}{$\begin{array}{c}\text { Horz } \\
2.00\end{array}$} & \multirow{2}{*}{$\frac{\text { Vert }}{2.25}$} & \multirow{2}{*}{$\frac{\text { 3D }}{3.25}$} \\
\hline$[1][2]$ & GPS $\quad C o$ & & & & \\
\hline \multirow[t]{2}{*}[1]{$[2]$} & \multirow{2}{*}{\multicolumn{2}{|c|}{$\begin{array}{l}\text { DGPS } \text { Code }^{1} \\
\text { Carrier }^{2}\end{array}$}} & 1.75 & 1.75 & 2.50 \\
\hline & & & 0.30 & 0.20 & 0.35 \\
\hline [4] & RRS & & 1.40 & 1.00 & 1.70 \\
\hline \multicolumn{6}{|c|}{ SRS Range Constraints: ${ }^{1} 300-500 \mathrm{~nm}{ }^{2} 50-100 \mathrm{~nm}$} \\
\hline \multirow{2}{*}{\multicolumn{2}{|c|}{$\begin{array}{l}\text { [ Subsystem ] } \\
\text { Configuration }\end{array}$}} & \multicolumn{4}{|c|}{ RMS Velocity (m/s) } \\
\hline & & East & North & Up & 3D \\
\hline [1] IN & S/EGI & 0.010 & 0.010 & 0.010 & 0.017 \\
\hline [3] IN & S/ESNU & 0.005 & 0.005 & 0.005 & 0.010 \\
\hline
\end{tabular}

Attitude Accuracy: 20 arcsec ( Roll,Pitch,Heading ) 


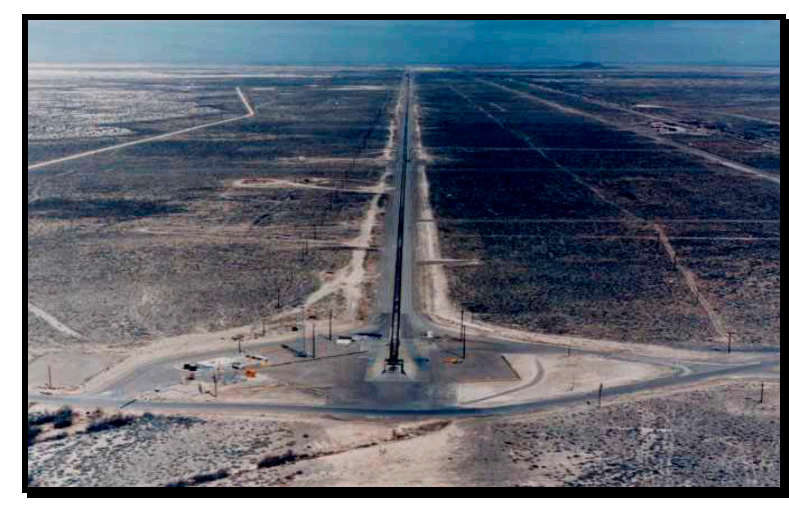

Figure 5: Holloman High-Speed Sled Track

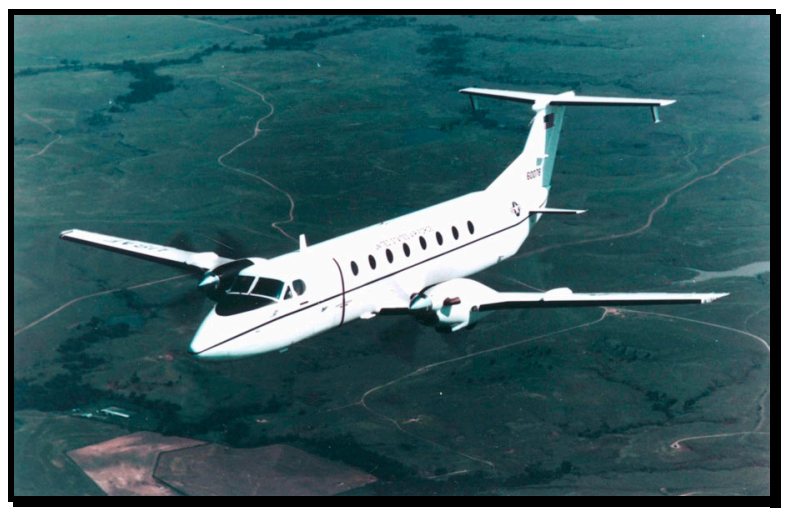

Figure 7: Aircraft (C-12J) Medium Dynamic

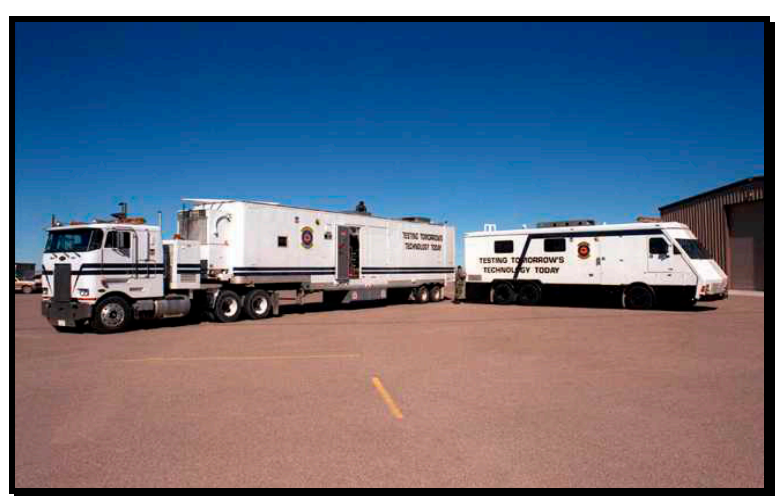

Figure 6: Vans (LNV) Low Dynamic

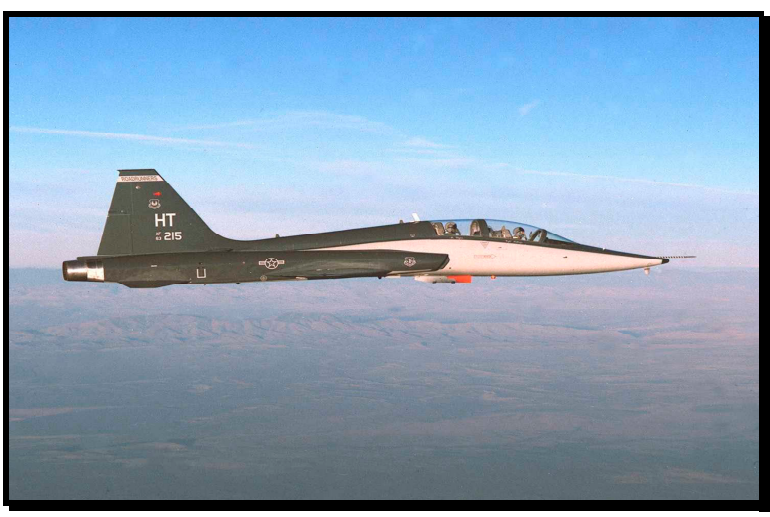

Figure 8: Aircraft (T-38) High Dynamic

\section{FUTURE DEVELOPMENT}

CRS is presently the primary reference system for van and flight tests at the 746th Test Squadron, with special requirements in the GPS jamming environment. Performance has been exceptional within the development design constraints. However, sub-meter position accuracy during high dynamic and GPS jamming environments is a future requirement.

The EGI subsystem GPS module has been updated to all-in-view and carrier phase measurement capability, which allows sub-meter position accuracy in a high dynamic environment. Presently the capability is being characterized for future test programs.

A future goal is replacement of the RSS (transponders/interrogators) subsystem, with a more accurate sub-meter system to increase the reference system performance in a GPS-jammed environment. 
The characterization and evaluation of state-of-the-art navigation systems require accurate reference systems. CRS, supporting a selectable subsystem configuration, was designed to test highly accurate navigation systems and possesses many unique capabilities, especially the ability to maintain sub-meter accuracy in high dynamic environment. However, the evolution of inertial components, aiding (GPS) and processing capabilities requires similar reference system advancements. Incorporating future improvements with present capabilities produces reference systems of the future.

\section{REFERENCES}

[1] Reference System Characterization Tests CHAPS Summary; 746th Test Squadron, 46th Test Group, Holloman AFB, NM; January 1997.

[2] Combining MOSES and PNAV Solutions; Carlson, N., Integrity Systems; Technical Memorandum MOSES-2000-05; April 2000.

[3] The CIGTF High Accuracy Post-processing Reference System (CHAPS); Raquet, J, 746th Test Squadron, Holloman AFB, NM; 1995.

[4] Enhanced Precision Reference System; Lawrence, R, 746th Test Squadron, Holloman AFB, NM; ION June 2001.

[5] Stochastic Models, Estimation, and Control Volume 2; Maybeck, P. Department of Electrical Engineering, Air Force Institute of Technology, Wright-Patterson AFB, OH; 1982. 